\title{
Effectiveness of focused extracorporeal shock wave therapy in the treatment of carpal tunnel syndrome
}

\author{
A randomized, placebo-controlled pilot study
}

\section{Christina Gesslbauer · Michael Mickel · Othmar Schuhfried · Dominikus Huber • Mohammad Keilani • Richard Crevenna (D)}

Received: 14 July 2020 / Accepted: 19 November 2020 / Published online: 22 December 2020

(c) The Author(s) 2020

\begin{abstract}
Summary
Background The carpal tunnel syndrome is the most common entrapment neuropathy in the general population. A conservative treatment should be considered in mild to moderate cases. The aim of this study was to assess the effect of a focused extracorporeal shock wave therapy in the treatment of mild to moderate carpal tunnel syndrome.

Material and Methods In this study 30 patients were randomly assigned into 2 groups. Subjects in the study group received three sessions of focused extracorporeal shock wave therapy, whereas the control group underwent a sham therapy. Patients were evaluated 3 and 12 weeks after treatment. The primary outcome was the visual analogue scale score. Secondary outcome measurements included hand grip strength, Boston Carpal Tunnel Syndrome Questionnaire, SF-36 Health Survey and electrodiagnostic measurements. Results A significant improvement of visual analogue scale at week $3(p=0.018)$ and week $12(p=0.007)$ as well as hand grip strength at week $12(p=0.019)$ could be observed in the study group. The study group showed a significantly better sensory nerve conduction velocity at week 12 than the control group, before correcting for multiple testing, and also a significant improvement in distal motor latency of the median nerve at week $12(p=0.009)$ as well as in both questionnaires (SF-36 subscale bodily pain, $p=0.020$ and severity symptom scale, $p=0.003$ ). No such improvement was observed in the control group.
\end{abstract}

C. Gesslbauer · M. Mickel · O. Schuhfried · D. Huber . M. Keilani · Univ.-Prof. Dr. R. Crevenna, MMSc, MBA $(\bowtie)$ Department of Physical Medicine, Rehabilitation and Occupational Medicine, Medical University of Vienna, Währinger Gürtel 18-20, 1090 Vienna, Austria richard.crevenna@meduniwien.ac.at
Conclusion Focused extracorporeal shock wave therapy is an effective and noninvasive treatment method for mild to moderate carpal tunnel syndrome.

Keywords Pain management - Conservative management $\cdot$ Median neuropathy $\cdot$ Night splint . Treatment outcome

\section{Introduction}

The carpal tunnel syndrome (CTS) is the most common entrapment neuropathy in the general population. It is a condition caused by compression of the median nerve. Most cases of CTS are idiopathic leading to chronic pressure increase and subsequently ischemia of the median nerve and segmental demyelination [1]. The CTS is more common among women as compared to men. Further risk factors include repetitive wrist movements, pregnancy, obesity, rheumatoid arthritis, diabetes mellitus and menopause. Symptoms usually start gradually at night and include paresthesia, burning and/or tingling in the territory of the median nerve as well as nocturnal pain and in severe cases also weakness of the hand and thenar atrophy [1]. In chronic and untreated cases CTS can lead to irreversible nerve damage. The diagnosis of CTS is usually clinical in a patient with characteristic symptoms and includes a physical examination and electrodiagnostic testing. There are different methods to treat CTS patients [1, 2]. A conservative treatment should be considered in mild to moderate cases and consists of splinting, physical modalities (e.g. therapeutic ultrasound, low level laser therapy), oral corticosteroids or nonsteroidal anti-inflammatory drugs. Other treatment options include steroid injection and surgery. In severe cases a surgical treatment should be considered [1-5]. Positive effects especially for wrist splint, local 
corticosteroid injection and surgical treatment have been demonstrated in multiple studies [1-5]; however, steroid injection and surgery always carry some risks of side effects (e.g. infections or allergic reactions). Extracorporeal shock wave therapy (ESWT) is a noninvasive and novel treatment option. It is based on the generation of acoustic waves which interact directly with cells by mechanotransduction by activating the metabolic rate which leads to tissue remodelling [6]. The ESWT can be classified into focused ESWT (fESWT) and radial ESWT (rESWT). While rESWT has a more superficial effect and reaches the maximum energy at the skin surface and distributes it radially into the tissue, fESWT develops the maximum energy at a focus located deeper in the body tissues [7]. Over the last years ESWT has gained widespread attention. Different types of diseases and conditions can be treated in a very effective and safe way without severe side effects $[8,9]$. It has been demonstrated to have anti-inflammatory, analgesic and proliferative effects [10-15] and it has also been proven to have effects for the reinnervation of peripheral nerves [16-18]. Therefore, both fESWT and rESWT, have received increased attention in the treatment of CTS and several studies have already yielded partially positive effects [19-23]. $\mathrm{Xu}$ et al. demonstrated significantly greater improvement in visual analogue scale (VAS) and the Boston carpal tunnel syndrome questionnaire (BCTQ) in the ESWT group compared to the local corticosteroid injection group [19]. Wu et al. reported positive results after a treatment with rEWST. They concluded that rESWT is a safe and effective method for relieving pain [20]. Similar effects were shown in a study of Vahatpour et al. [21]. Because ESWT is a novel treatment there is still little known about the efficacy, the long-term effects and adverse events of ESWT in the treatment of CTS. Hence, the aim of this study was to assess the effect of fESWT in the treatment of mild to moderate carpal tunnel syndrome.

\section{Materials and Methods}

\section{Trial design and participants}

This pilot study was a randomized, single-blinded, placebo-controlled pilot study.

The project was approved by the ethics committee of the Medical University of Vienna (EK Nr. 1080/2019) and all subjects gave written informed consent to participate. The study conformed to the principles of the Declaration of Helsinki. There was no commercial sponsorship.

Inclusion criteria were mild to moderate CTS objectively verified using electrodiagnostic testing [24, 25]. Exclusion criteria were metabolic diseases, blood clotting disorders, systemic diseases, polyneuropathy, chemotherapy during the study, corticosteroid therapy, use of anticoagulation, history of trauma/ surgery or nerve lesions of the treated extremity,
CTS surgery on the affected hand, implantable cardioverter defibrillator (ICD)/pacemaker implantation, other therapy for the affected hand during the study, acute inflammation or infections, severe mental illnesses/psychiatric diseases, and severe neurological diseases.

Patients from the outpatient clinic (Department of Physical Medicine, Rehabilitation and Occupational Medicine) who met the inclusion criteria and who were diagnosed with mild to moderate CTS by electrodiagnostic testing were randomized to receive either fESWT or sham fESWT. A total of 30 patients were enrolled, all of which participated in the determination of the baseline measurements. Of these 20 patients proceeded to receive treatment and the follow-up examinations, 4 of the patients dropped out for personal reasons, while the remaining 6 drop-outs could not complete the trial due to coronavirus disease 2019 (COVID-19) restrictions on visits to the clinic. The results were analyzed on a per protocol basis.

In the study group (fESWT group), participants received weekly fESWT for 3 consecutive weeks. In the control group the participants received sham fESWT for the same interval. Additionally, all subjects were asked to wear night splints. Outcome measure were the VAS [26], hand grip strength (using a Jamar hand grip dynamometer [JAMAR ${ }^{\circledR}$ dynamometry (Patterson Medical, Warrenville, IL, USA)] [27]) and electrodiagnostic parameters (distal motor latency and sensory nerve conduction velocity) [24, 25]. Subjects were also tested with questionnaires by using the SF-36 Health Survey (SF-36) [28] and the BCTQ [29, 30]. All patients were evaluated at baseline (T0: VAS, hand grip, electrodiagnostic parameters, questionnaires), week 3 (T1: VAS, hand grip, questionnaires), and week 12 (T2: VAS, hand grip, electrodiagnostic parameters, questionnaires) after treatment by the same physician. Of the patients 20 have completed the 3 -month evaluation. No adverse effects occurred.

\section{Randomization and blinding}

Randomization was performed by using sequentially numbered sealed envelopes. Eligible participants were then randomly assigned to either the fESWT group (intervention group) or the control group (sham fESWT). The participants and the investigator who evaluated the baseline/outcome measures were blinded with respect to the group allocation.

\section{Intervention}

Patients in the fESWT group were treated three times with fESWT (PiezoWave2, Richard Wolf GmbH, Knittlingen, Germany) (Fig. 1). After identifying the carpal tunnel area by using musculoskeletal ultrasonography (ECUBE i7, Alpinion Medical Systems, Seoul, Korea) fESWT was applied to the flexor retinaculum (transverse carpal ligament) by using a linear therapy 
Fig. 1 Application of focused extracorporeal shock wave therapy

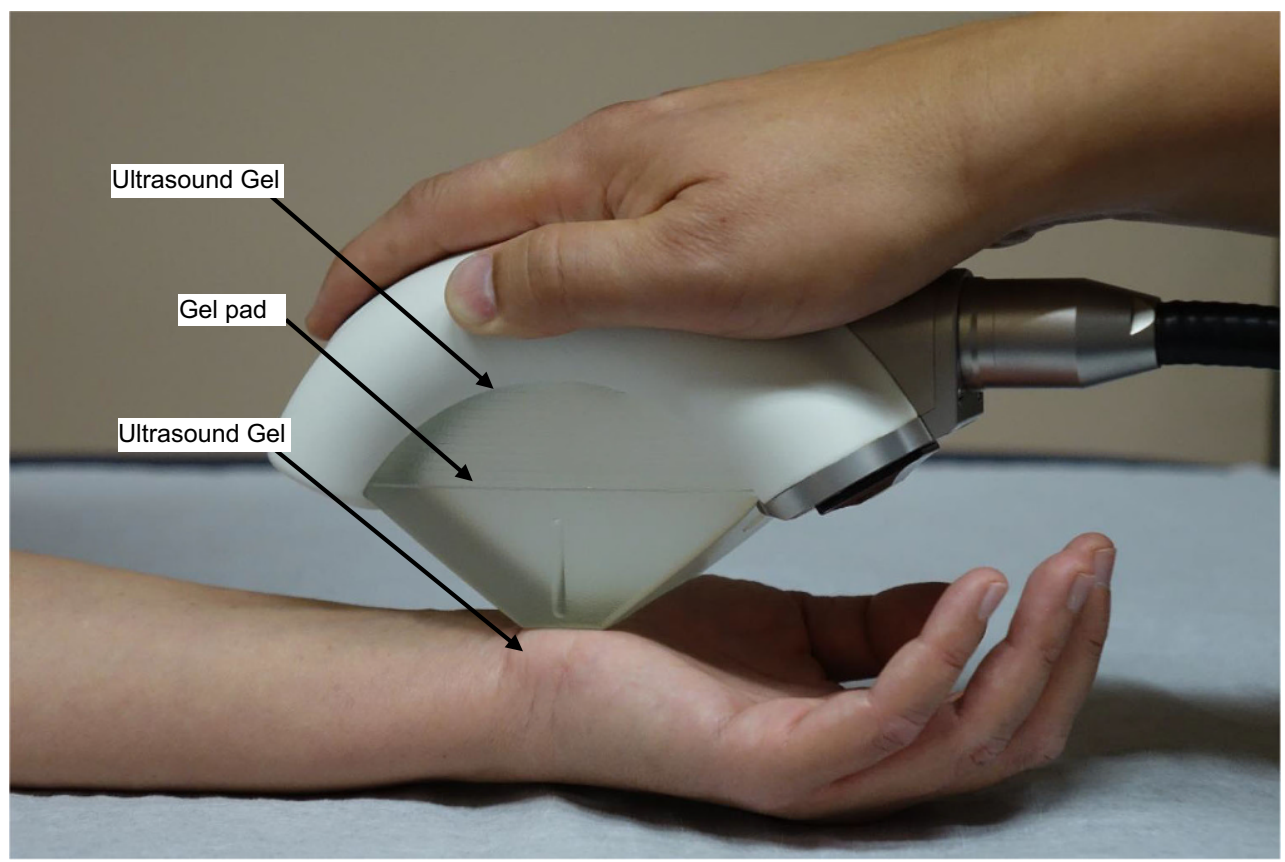

source. Each patient received a linear fESWT (therapy source FBL 10X5G2, PiezoWave2) that comprised 500 shocks at energy flux density (EFD) of $0.05 \mathrm{~mJ} / \mathrm{mm}^{2}$ (maximum). The pulse repetition frequency was $4 \mathrm{~Hz}$. In the fESWT group, a three-layered coupling medium between the applicator head and the tissue/skin was used (ultrasound gel-gel pad-ultrasound gel) to efficiently transduce the shock wave into the tissue (Fig. 1). In the control group only a two-layered coupling medium was used and the ultrasound gel layer between applicator head and gel pad was omitted to achieve a placebo treatment effect. The treatment process was identical to that of the fESWT group, with the same sound signals during the procedure. Since there is still no standardized treatment recommendation of fESWT for CTS we chose to perform the fESWT/sham fESWT once a week for a period of 3 weeks by the same physician. Additionally, all subjects were asked to wear night splints.

\section{Outcome measures}

All patients were evaluated at baseline (T0: VAS, hand grip, electrodiagnostic parameters, questionnaires) week 3 (T1: VAS, hand grip, questionnaires), and week 12 (T2: VAS, hand grip, electrodiagnostic parameters, questionnaires).

\section{Primary outcome measure}

1. The subjective pain intensity was measured with a $100 \mathrm{~mm}$ VAS, 0 indicating no pain and 100 the strongest imaginable pain [25].

\section{Secondary outcomes}

2. The distal motor latency of the median nerve (DML) and antidromic sensory conduction velocity of the median nerve (SNCV) were evaluated by using NCV (Keypoint device, Medtronic, Dantec Medical A/S, Skovlunde, Denmark) [24, 25]. The ulnar nerve was also evaluated to exclude other peripheral neuropathies. All evaluations were executed in the same room by the same physiatrist.

3. The hand grip strength was evaluated using a hand grip dynamometer [28].

4. The 36-item Short Form Health Survey questionnaire (SF-36) consists of 36-items and 8 scales and measures the physical and mental health [29].

5. The BCTQ evaluates the severity of symptoms and the functional status of patients with carpal tunnel syndrome. The symptom severity scale (SSS) consists of 11 questions covering symptom severity and scores from 1 point (mildest) to 5 points (most severe). The functional status scale (FSS) consists of 8 points ranging from 1 point (no difficulty with the activity) to 5 points (cannot perform the activity at all) $[29,30]$.

\section{Statistical analysis}

Statistical analysis was carried out using GraphPad Prism 8 software (GraphPad, San Diego, CA, USA).

Testing for normality was assessed groupwise via the Shapiro-Wilk test. When employing an ANOVA, sphericity was not assumed and therefore not tested for. No significant outliers in the data analyzed via ANOVA were observed. Sample size was computed with a power of 0.80 . 
A $p$-value $<0.05$ was considered as statistically significant. The $95 \%$ confidence interval for mean differences is reported in brackets next to the value.

If not specified otherwise, the Holm-Sidak method for correcting for multiple testing was applied to all pairwise comparisons and adjusted $p$-values reported accordingly.

\section{Results}

\section{Patients}

A total of 20 patients completed the 3month followup. Table 1 shows the baseline demographic data and clinical characteristics of patients who completed the

Table 1 Demographic data for both drop-outs and included groups. A Holm-Sidak test showed no significant difference between these groups regarding the demographics

\begin{tabular}{|c|c|c|c|c|c|c|}
\hline & \multicolumn{2}{|c|}{ fESWT group } & \multicolumn{2}{|c|}{ Control group } & \multicolumn{2}{|c|}{ Drop-outs } \\
\hline & Mean & SD & Mean & SD & Mean & SD \\
\hline Age (years) & 55.80 & 4.66 & 54 & 17.40 & 53.10 & 7.48 \\
\hline Female sex, $n(\%)$ & 8 & $80 \%$ & 6 & $60 \%$ & 7 & $70 \%$ \\
\hline Height (cm) & 167.80 & 6.61 & 169.70 & 11.81 & 171.40 & 11.07 \\
\hline Weight (kg) & 81.10 & 18.91 & 87.40 & 29.72 & 71.30 & 7.53 \\
\hline $\begin{array}{l}\text { Hoffman-Tinel } \\
\text { sign, } n(\%)\end{array}$ & 1.40 & 0.52 & 1.30 & 0.48 & 0.80 & 0.42 \\
\hline $\begin{array}{l}\text { Duration of symp- } \\
\text { toms (months) }\end{array}$ & 29 & 32.89 & 33.60 & 44.26 & 40.80 & 40.77 \\
\hline
\end{tabular}

study. The mean age in the fESWT group ( $n=10)$ was $55.8 \pm 4.6$ years. They experienced CTS symptoms for a mean of $29.0 \pm 32.8$ months.

The mean age in the control group $(n=10)$ was $54.0 \pm 17.4$ years. They experienced CTS symptoms for a mean of $33.6 \pm 44.2$ months.

$T$-tests for multiple comparisons between the fESWT, the control, and the drop-out group for all main outcome variables showed no significant differences in baseline measurements (Tables 2, 3 and 4). Table 5 gives an overview of all outcome measures at each point in time.

\section{Intergroup comparison}

Multiple $t$-tests showed no statistically significant difference between the fESWT and the control group at T1 (Table 6). At T2, the fESWT group did significantly better regarding SNCV (mean difference $=5.0$, SE of difference $=2.0, p=0.02$ ) before correcting for multiple testing (Table 7). After correction via the HolmSidak method, however, there was no significant difference between groups.

\section{ANOVA by time and group}

VAS

In absolute values, VAS decreased over time in the fESWT group. The control group showed no clear direction of VAS over time (Fig. 2). A two-way repeated measurements ANOVA for pain (VAS) at T0, $\mathrm{T} 1$ and $\mathrm{T} 2$ for both groups showed a significant effect

Table 2 Main outcome parameters fESWT vs. control group at TO

\begin{tabular}{|c|c|c|c|c|c|c|c|}
\hline & $P$-value & $\begin{array}{l}\text { Mean of fESWT } \\
\text { group }\end{array}$ & $\begin{array}{l}\text { Mean of control } \\
\text { group }\end{array}$ & Difference & SE of difference & t ratio & Adjusted $p$-value \\
\hline VAS & 0.49 & 50.5 & 58.3 & -7.80 & 10.96 & 0.71 & 0.98 \\
\hline Hand grip (kg) & 0.54 & 29.9 & 27.3 & 2.60 & 4.15 & 0.63 & 0.98 \\
\hline DML (ms) & 0.58 & 4.88 & 4.76 & 0.12 & 0.21 & 0.56 & 0.98 \\
\hline SNCV (m/s) & 0.18 & 40.2 & 38.35 & 1.85 & 1.32 & 1.41 & 0.74 \\
\hline SSS & 0.92 & 2.41 & 2.44 & -0.04 & 0.34 & 0.11 & $>0.99$ \\
\hline FSS & 0.88 & 1.73 & 1.79 & -0.06 & 0.42 & 0.15 & $>0.99$ \\
\hline SF-36 PAIN & 0.95 & 47.9 & 48.4 & -0.50 & 8.60 & 0.06 & $>0.99$ \\
\hline
\end{tabular}

VAS Visual analogue scale, DML Distal motor latency, SNCV Sensory nerve conduction velocity, SSS Symptom severity scale, FSS Functional status scale, SF36 PAIN Short Form 36 Health Survey, fESWT focused extracorporeal shock wave therapy, SE standard error

Table 3 Main outcome parameters fESWT vs. drop-out group at T0

\begin{tabular}{|c|c|c|c|c|c|c|c|}
\hline & $P$ value & $\begin{array}{l}\text { Mean of fESWT } \\
\text { group }\end{array}$ & Mean of drop-outs & Difference & SE of difference & t ratio & Adjusted $p$-value \\
\hline VAS & 0.76 & 50.5 & 47.5 & 3.00 & 9.83 & 0.31 & 0.98 \\
\hline Hand grip (kg) & 0.70 & 29.9 & 28.6 & 1.30 & 3.36 & 0.39 & 0.98 \\
\hline $\mathrm{DML}(\mathrm{ms})$ & 0.63 & 4.88 & 4.989 & -0.11 & 0.22 & 0.49 & 0.98 \\
\hline $\mathrm{SNCV}(\mathrm{m} / \mathrm{s})$ & 0.34 & 40.2 & 72.89 & -32.69 & 33.38 & 0.98 & 0.88 \\
\hline SSS & 0.95 & 2.409 & 2.431 & -0.02 & 0.39 & 0.06 & 0.98 \\
\hline FSS & 0.19 & 1.725 & 2.172 & -0.45 & 0.32 & 1.38 & 0.76 \\
\hline SF-36 PAIN & 0.21 & 47.9 & 36.8 & 11.10 & 8.57 & 1.30 & 0.76 \\
\hline
\end{tabular}


Table 4 Main outcome parameters control vs. drop-out group at T0

\begin{tabular}{|c|c|c|c|c|c|c|c|}
\hline & $P$-value & $\begin{array}{l}\text { Mean of control } \\
\text { group }\end{array}$ & Mean of drop-outs & Difference & SE of difference & t ratio & Adjusted $p$-value \\
\hline VAS & 0.35 & 58.3 & 47.50 & 10.80 & 11.21 & 0.96 & 0.85 \\
\hline Hand grip (kg) & 0.72 & 27.3 & 28.60 & -1.30 & 3.54 & 0.37 & 0.92 \\
\hline $\mathrm{DML}(\mathrm{ms})$ & 0.20 & 4.76 & 4.99 & -0.23 & 0.17 & 1.34 & 0.73 \\
\hline $\mathrm{SNCV}(\mathrm{m} / \mathrm{s})$ & 0.32 & 38.35 & 72.89 & -34.54 & 33.36 & 1.04 & 0.85 \\
\hline SSS & 0.98 & 2.445 & 2.43 & 0.01 & 0.46 & 0.03 & 0.98 \\
\hline FSS & 0.40 & 1.788 & 2.17 & -0.38 & 0.45 & 0.86 & 0.85 \\
\hline SF-36 PAIN & 0.15 & 48.4 & 36.80 & 11.60 & 7.63 & 1.52 & 0.67 \\
\hline
\end{tabular}

Table 5 Mean, standard deviation, and confidence interval of the mean for all outcome variables at each point of measurement

\begin{tabular}{|c|c|c|c|c|c|c|c|c|c|c|}
\hline & \multicolumn{5}{|c|}{ fESWT group } & \multicolumn{5}{|c|}{ Control group } \\
\hline & Mean & $\begin{array}{l}\text { Std. devia- } \\
\text { tion }\end{array}$ & $\begin{array}{l}\text { Std. error } \\
\text { of mean }\end{array}$ & $\begin{array}{l}\text { Lower } 95 \% \\
\text { Cl of mean }\end{array}$ & $\begin{array}{l}\text { Upper } 95 \% \\
\text { Cl of mean }\end{array}$ & Mean & Std. deviation & $\begin{array}{l}\text { Std. error of } \\
\text { mean }\end{array}$ & $\begin{array}{l}\text { Lower 95\% } \\
\text { Cl of mean }\end{array}$ & $\begin{array}{l}\text { Upper } 95 \% \\
\text { Cl of mean }\end{array}$ \\
\hline VAS TO & 51 & 21 & 6.7 & 35 & 66 & 58 & 27 & 8.6 & 39 & 78 \\
\hline VAS T1 & 31 & 25 & 7.8 & 13 & 48 & 42 & 25 & 7.8 & 24 & 59 \\
\hline VAS T2 & 28 & 23 & 7.4 & 11 & 44 & 46 & 24 & 7.7 & 28 & 63 \\
\hline Hand grip T0 (kg) & 30 & 8.9 & 2.8 & 23 & 36 & 27 & 9.6 & 3 & 20 & 34 \\
\hline Hand grip T1 (kg) & 32 & 10 & 3.2 & 24 & 39 & 29 & 6.7 & 2.1 & 24 & 34 \\
\hline Hand grip T2 (kg) & 34 & 9.7 & 3.1 & 27 & 41 & 28 & 8 & 2.5 & 22 & 33 \\
\hline DML T0 (ms) & 4.9 & 0.57 & 0.18 & 4.5 & 5.3 & 4.8 & 0.37 & 0.12 & 4.5 & 5 \\
\hline DML T2 (ms) & 4.5 & 0.64 & 0.2 & 4.1 & 5 & 4.5 & 0.6 & 0.19 & 4.1 & 5 \\
\hline SNCV T0 (m/s) & 40 & 3.9 & 1.2 & 37 & 43 & 38 & 1.5 & 0.46 & 37 & 39 \\
\hline SNCV T2 (m/s) & 44 & 5.7 & 1.8 & 40 & 48 & 39 & 2.8 & 0.9 & 37 & 41 \\
\hline SF-36 PAIN T0 & 48 & 21 & 6.7 & 33 & 63 & 48 & 17 & 5.4 & 36 & 61 \\
\hline SF-36 PAIN T1 & 58 & 14 & 4.4 & 48 & 68 & 44 & 23 & 7.2 & 28 & 60 \\
\hline SF-36 PAIN T2 & 54 & 24 & 7.5 & 37 & 71 & 47 & 22 & 7.1 & 31 & 63 \\
\hline SSS TO & 2.4 & 0.58 & 0.18 & 2 & 2.8 & 2.4 & 0.9 & 0.28 & 1.8 & 3.1 \\
\hline SSS T1 & 1.7 & 0.36 & 0.12 & 1.4 & 1.9 & 2.1 & 0.86 & 0.27 & 1.5 & 2.7 \\
\hline SSS T2 & 1.9 & 0.95 & 0.3 & 1.2 & 2.6 & 1.8 & 0.67 & 0.21 & 1.3 & 2.3 \\
\hline FSS T0 & 1.7 & 0.71 & 0.23 & 1.2 & 2.2 & 1.8 & 1.1 & 0.36 & 0.98 & 2.6 \\
\hline FSS T1 & 1.5 & 0.6 & 0.19 & 1 & 1.9 & 1.6 & 0.7 & 0.22 & 1.1 & 2.1 \\
\hline FSS T2 & 1.6 & 0.8 & 0.25 & 0.97 & 2.1 & 1.7 & 0.82 & 0.26 & 1.1 & 2.2 \\
\hline
\end{tabular}

of time $(p<0.001)$ and no significant effect of treatment group $(p=0.216)$ or the interaction of the two $(p=0.477)$. Multiple comparisons corrected by Dunnet's test revealed a significant difference of VAS between both T0 and T1 (mean difference $=19.9,95 \%$ confidence interval $[3.93 ; 35.9], p=0.018)$ and T0 and T2 (mean difference $=23.0,95 \%$ confidence interval [7.51; 38.5], $p=0.007$ ) in the fESWT group (Table 8; Fig. 2). In the control group, no significant difference could be observed (mean difference T0 vs. $\mathrm{T} 1=16.8$, $95 \%$ confidence interval $[-0.761 ; 34.4], p=0.059$; mean difference T0 vs. $\mathrm{T} 2=12.5,95 \%$ confidence interval $[-4.56 ; 29.6], p=0.151)$.

\section{Hand grip}

The two-way repeated measurements ANOVA for hand grip strength at $\mathrm{T} 0, \mathrm{~T} 1$ and $\mathrm{T} 2$ yielded no significant effect of any kind (time: $p=0.108$; group: $p=0.330$; time ${ }^{*}$ group: $p=0.140$ ) [The interaction term time ${ }^{*}$ group describes the effect we are interested in, i.e. the effect on patients in the fESWT (group) group after intervention (time)]. Dunnet's multiple comparisons test, however, revealed a significant improvement on baseline hand grip strength at T2 in the fESWT group (mean difference T2 vs. $\mathrm{T} 0=3.90$ [0.72; 7.08], $p=0.019$ ) (Table 8; Fig. 3). 
Table 6 Differences in outcome measures at T1 corrected via the Holm-Sidak method for multiple testing

\begin{tabular}{|c|c|c|c|c|c|c|c|}
\hline $\mathrm{T} 1$ & $P$-value & $\begin{array}{l}\text { Mean of fESWT } \\
\text { group }\end{array}$ & Mean of control group & Difference & SE of difference & t ratio & Adjusted $p$-value \\
\hline VAS & 0.34 & 30.6 & 41.5 & -10.90 & 11.02 & 0.99 & 0.71 \\
\hline Hand grip (kg) & 0.5 & 31.6 & 29 & 2.60 & 3.80 & 0.69 & 0.75 \\
\hline SSS & 0.15 & 1.66 & 2.11 & -0.45 & 0.30 & 1.51 & 0.48 \\
\hline FSS & 0.58 & 1.46 & 1.63 & -0.16 & 0.29 & 0.56 & 0.75 \\
\hline SF-36 PAIN & 0.12 & 58.1 & 44.3 & 13.80 & 8.37 & 1.65 & 0.46 \\
\hline
\end{tabular}

VAS Visual analogue scale, SSS Symptom severity scale, FSS Functional status scale, SF-36 PAIN Short Form 36 Health Survey, fESWT focused extracorporeal shock wave therapy, $S E$ standard error

Table 7 Differences in outcome measures at T2 corrected via the Holm-Sidak method for multiple testing

\begin{tabular}{|c|c|c|c|c|c|c|c|}
\hline T2 & $P$-value & $\begin{array}{l}\text { Mean of fESWT } \\
\text { group }\end{array}$ & $\begin{array}{l}\text { Mean of control } \\
\text { group }\end{array}$ & Difference & SE of difference & t ratio & Adjusted $p$-value \\
\hline VAS & 0.1 & 27.5 & 45.8 & -18.30 & 10.64 & 1.72 & 0.48 \\
\hline Hand grip (kg) & 0.14 & 33.8 & 27.6 & 6.20 & 3.98 & 1.56 & 0.52 \\
\hline DML (ms) & 0.94 & 4.52 & 4.54 & -0.02 & 0.28 & 0.07 & 0.98 \\
\hline SNCV (m/s) & 0.02 & 43.75 & 38.75 & 5.00 & 2.01 & 2.48 & 0.15 \\
\hline SSS & 0.71 & 1.93 & 1.79 & 0.14 & 0.37 & 0.37 & 0.98 \\
\hline FSS & 0.76 & 1.55 & 1.66 & -0.11 & 0.36 & 0.31 & 0.98 \\
\hline SF-36 PAIN & 0.49 & 53.9 & 46.6 & 7.30 & 10.28 & 0.71 & 0.93 \\
\hline
\end{tabular}

95\% Confidence Intervals (Dunnett)

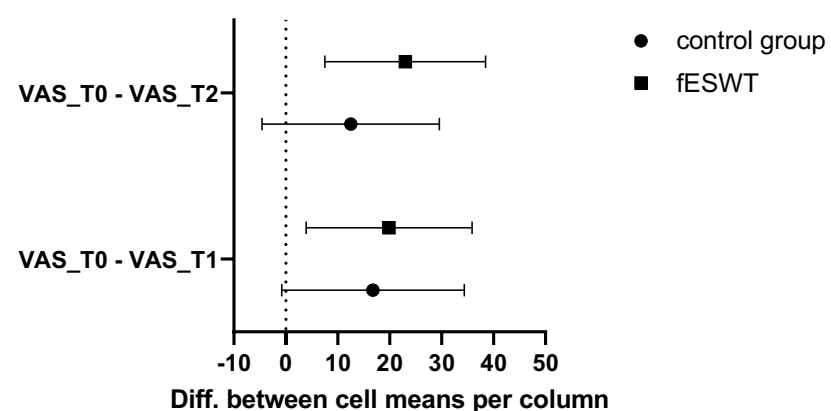

Fig. 2 Confidence intervals for difference in pain (cell means) between baseline (T0) vs. posttreatment (T1) (bottom) and baseline vs. 3-month follow-up (T2) (top). There is a significant improvement in pain only in the verum group. Despite a visible trend in T2 vs. T0, no difference could be detected between the two treatment groups (columns)

\section{Electroneurography}

Due to the naturally high correlation of these variables, a two-way repeated-measurements ANOVA was carried out for SNCV and DML simultaneously. The analysis of variance yielded significant effects of time $(p<0.001)$, treatment group $(p=0.024)$ and their interaction $(p=0.010)$. Corrected via Tukey's test for multiple comparisons, a significant improvement of DML (mean difference $=-0.36,95 \%$ confidence interval $[-0.62 ;-0.10], p=0.009$ ) but no significant effect for sNCV (mean difference $=3.55$, 95\% confidence interval $[-0.450 ; 7.55], p=0.084)$ was observed in the fESWT group (Table 8).

\section{SF-36}

The SF-36 subscale bodily pain was compared at all three points of measurement $\mathrm{T} 0, \mathrm{~T} 1$, and $\mathrm{T} 2$ via twoway repeated measurements ANOVA. Time $(p=0.515)$ and treatment group $(p=0.431)$ showed no significant effect on pain scoring but their interaction did $(p=0.049)$. Dunnet's test for multiple comparisons showed a significant effect on the SF-36 pain score at T0 compared to T1 in the fESWT group (mean difference $=-10.2,95 \%$ confidence interval $[-18.6 ;-1.81]$, $P=0.020$ ) (Table 8 ), but no other significant results.

\section{Boston carpal tunnel syndrome questionnaire}

A two-way repeated-measurements ANOVA at T0, T1 and T2 for the SSS suggested a significant effect of time ( $p=0.002)$, while treatment group $(p=0.681)$ and time * group $(p=0.213)$ showed no significant difference. A pairwise comparison using Dunnet's test revealed a significant difference between SSS at T0 and T1 (mean difference $=0.74,95 \%$ confidence interval $[0.31 ; 1.18], p=0.003$ ) but no other differences (Table 8; Fig. 4). Generally, participants in both groups saw a small increase of their scores over time.

\section{Discussion}

The aim of this randomized, single blinded, placebocontrolled pilot study was to investigate the effect of three sessions of fESWT in patients with mild to moderate CTS. Our results strengthen the therapeutic effects of (f)ESWT once more. Compared to the control group, a significant improvement of VAS in the 
original article

Table 8 Pair-wise comparison of all outcome parameters after carrying out a repeated-measures two-way ANOVA (Analysis of variance) for the three time points T0, T1 and T2 for the respective variable

\begin{tabular}{|c|c|c|c|}
\hline Outcome parameter/test & Mean diff & 95.\% $\mathrm{Cl}$ of diff & Adjusted $p$-value \\
\hline \multicolumn{4}{|l|}{ (Dunnet's) } \\
\hline \multicolumn{4}{|l|}{ Control group } \\
\hline VAS_T0 vs. VAS_T1 & 16.8 & -0.76 to 34.36 & 0.0599 \\
\hline VAS_T0 vs. VAS_T2 & 12.5 & -4.56 to 29.56 & 0.1504 \\
\hline \multicolumn{4}{|l|}{ fESWT group } \\
\hline VAS_T0 vs. VAS_T1 & 19.9 & 3.92 to 35.87 & 0.018 \\
\hline VAS_T0 vs. VAS_T2 & 23 & 7.51 to 38.49 & 0.0069 \\
\hline \multicolumn{4}{|l|}{ (Dunnet's) } \\
\hline \multicolumn{4}{|l|}{ Control group } \\
\hline Hand-grip strength T0 vs. T1 & -1.7 & -6.65 to 3.25 & 0.5893 \\
\hline Hand-grip strength T0 vs. T2 & -0.3 & -3.98 to 3.38 & 0.9673 \\
\hline \multicolumn{4}{|l|}{ fESWT group } \\
\hline Hand-grip strength T0 vs. T1 & -1.7 & -4.46 to 1.06 & 0.2363 \\
\hline Hand-grip strength T0 vs. T2 & -3.9 & -7.08 to -0.72 & 0.0194 \\
\hline \multicolumn{4}{|l|}{ (Tukey's) } \\
\hline \multicolumn{4}{|l|}{ Control group } \\
\hline DML T0 vs. SNCV TO & -33.59 & -35.25 to -31.93 & $<0.0001$ \\
\hline DML T0 vs. T2 & 0.22 & -0.08 to 0.52 & 0.1666 \\
\hline DML T0 vs. SNCV T2 & -33.99 & -37.07 to -30.91 & $<0.0001$ \\
\hline SNCV TO vs. DML T2 & 33.81 & 31.90 to 35.72 & $<0.0001$ \\
\hline SNCV T0 vs. T2 & -0.4 & -2.56 to 1.76 & 0.9358 \\
\hline DML T2 vs. SNCV T2 & -34.21 & -37.52 to -30.90 & $<0.0001$ \\
\hline \multicolumn{4}{|l|}{ fESWT group } \\
\hline DML T0 vs. SNCV TO & -35.32 & -39.34 to -31.30 & $<0.0001$ \\
\hline DML T0 vs. T2 & 0.36 & 0.09 to 0.62 & 0.0094 \\
\hline DML T0 vs. SNCV T2 & -38.87 & -44.80 to -32.94 & $<0.0001$ \\
\hline SNCV T0 vs. DML T2 & 35.68 & 31.57 to 39.79 & $<0.0001$ \\
\hline SNCV T0 vs. T2 & -3.55 & -7.55 to 0.45 & 0.0847 \\
\hline DML T2 vs. SNCV T2 & -39.23 & -45.29 to -33.17 & $<0.0001$ \\
\hline \multicolumn{4}{|l|}{ (Dunnet's) } \\
\hline \multicolumn{4}{|l|}{ fESWT group } \\
\hline SF36 pain T0 vs. T1 & -10.2 & -18.59 to -1.81 & 0.0204 \\
\hline SF36 pain T0 vs. T2 & -6 & -20.88 to 8.89 & 0.4954 \\
\hline \multicolumn{4}{|l|}{ Control group } \\
\hline SF36 pain T0 vs. T1 & 4.1 & -3.95 to 12.15 & 0.349 \\
\hline SF36 pain T0 vs. T2 & 1.8 & -6.96 to 10.56 & 0.8169 \\
\hline \multicolumn{4}{|l|}{ (Dunnet's) } \\
\hline \multicolumn{4}{|l|}{ fESWT group } \\
\hline SSS T0 vs. SSS T1 & 0.7454 & 0.31 to 1.18 & 0.0028 \\
\hline SSS T0 vs. SSS T2 & 0.4819 & -0.09 to 1.06 & 0.1016 \\
\hline \multicolumn{4}{|l|}{ Control group } \\
\hline SSS T0 vs. SSS T1 & 0.3365 & -0.45 to 1.13 & 0.4636 \\
\hline SSS T0 vs. SSS T2 & 0.6547 & 0.02 to 1.33 & 0.0551 \\
\hline
\end{tabular}


Fig. 3 Differences in hand grip strength at T0 vs. T1 and TO vs. T2 in both fESWT and control group showed a tendency of improvement

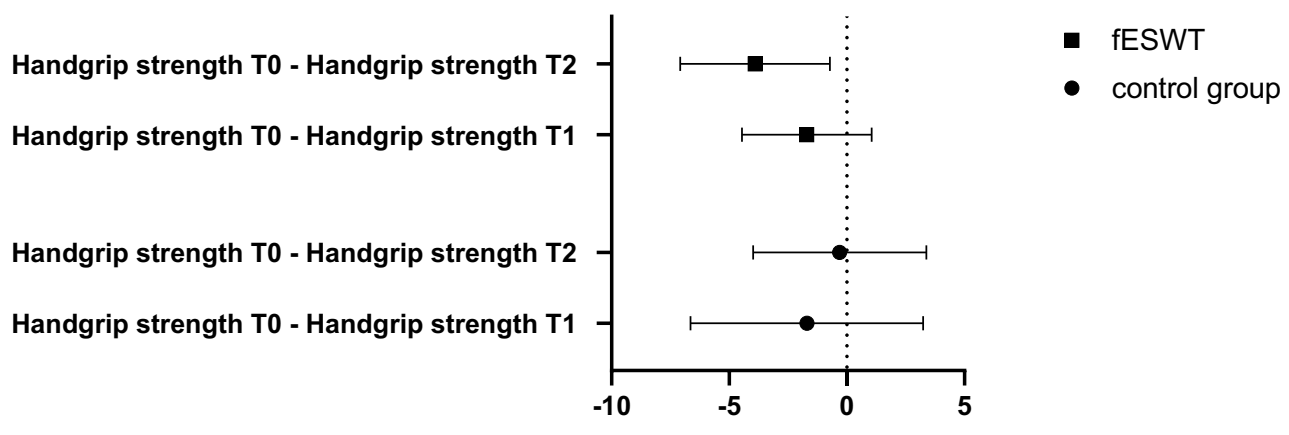

Diff. between cell means per column
95\% Confidence Intervals (Dunnett)

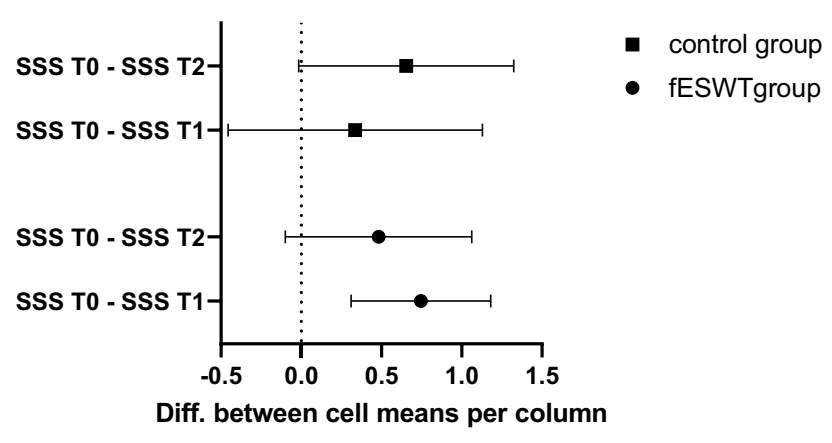

Fig. 4 Differences in Boston carpal tunnel syndrome questionnaire (symptom severity scale) at T0 vs. T1 and T0 vs. T2 showed a significant short-term improvement in the fESWT group

fEWST group appeared after three sessions of fESWT. The trend in the follow-up after 3 months showed a further significant improvement of VAS and a significant improvement of hand grip strength in the fESWT group. Concerning electrodiagnostic parameters, our study found a significant decrease of DML in the fESWT group at week 12 compared to baseline while the other electrodiagnostic parameter (sNCV) did not show any significant improvement between baseline and week 12 although there was a greater difference in the fESWT group for sNCV compared to the control group. Considering the SF36 questionnaire, improvement was observed for the subscale bodily pain at week 12 in the fESWT group. These results are in accordance with the results of VAS.

The CTS is the most common form of median nerve entrapment. In mild to moderate cases a conservative treatment should be considered. The use of ESWT for CTS is a novel treatment method and growing data have shown that ESWT is an effective and potential treatment option [11, 12, 14, 19-23]. Seok et al. administered one session of fESWT to treat patients with CTS and compared them to patients after corticosteroid injection. They found significant reductions in VAS and BCTQ scores in both groups at 1 and 3 months follow-up. Mild improvement was noted in the fESWT group for nerve conduction parameters. The authors concluded that ESWT could be as useful as corticosteroid injections [14]. Paoloni et al. reported that patients with mild to moderate CTS might experience pain relief and increased functionality after three sessions of fESWT compared to US and cyro ultrasound therapy [12]. In a study of Wu et al. the intervention group received three session of rESWT with night splinting, whereas the control group received sham rESWT and night splinting. A significantly greater improvement in VAS, BCTQ and crosssectional area (CSA) of the median nerve was shown in the intervention group. They concluded that rESWT is an effective method for pain relief [20]; however, the exact biological effects of ESWT still remain unknown and the definitive mechanism of ESWT on peripheral nerves/neuropathy is unclear. It has been demonstrated that ESWT seems to stimulate the production of endothelial nitric oxide (NO), angiogenesis and neurogenesis through vascular endothelial growth factor (VEGF), has anti-inflammatory effects through reduction in the release of calcitonin gene-related peptide (CGRP) and has also neuronal regeneration effects and promotes axonal regeneration of peripheral nerves through molecular reactions [13, 31-34]. Shock waves also stimulate tenocyte proliferation and collagen synthesis [35, 36]. In 2001 Ohtori et al. concluded that shock wave application to rat skin causes reinnervation of sensory nerve fibers [16]. Hausner et al. investigated whether ESWT improves the regeneration of injured nerves in an experimental rat model and proved that ESWT is effective in promoting axonal regeneration [17]. Mense et al. reported similar findings [18]. Very interesting are also the findings of Miyamoto et al. who compared the elasticity and thickness of the transverse carpal ligament by sonoelastography. Their results showed that the transverse carpal ligament was significantly thicker and harder in CTS patients and concluded that an increased stiffness could be one of the reasons for CTS [37]. In summary the anti-inflammatory, antinociceptive, neuronal regeneration effects as well as the apparent influence of the extracellular matrix might be the main mechanism for CTS improvement when 
using fESWT. It is possible that the anti-inflammatory effect can reduce the perineural pressure and neuronal regeneration may lead to an improvement of the electrodiagnostic parameters. Moreover, we hypothesize that the treatment with linear focused shock waves may stimulate the elasticity of the transverse carpal ligament via mechanotransduction effects and therefore relieve the CTS symptoms. Hence, it would be interesting for future studies to investigate if morphological changes of the median nerve also occur during/after fESWT.

There were some limitations of this study, most importantly the low number of participants and the high number of drop-outs. This limitation was largely due to the sudden closure of facilities due to COVID19. Although no significant differences in baseline measurements between the drop-outs and any of the groups could be observed, the per protocol analysis and power could be improved in future studies. Furthermore, our findings indicate that the long-term results at both 3 months and longer intervals of followup examinations deserve a closer look when assessing the efficacy of ESWT.

\section{Conclusion}

Our results suggest that fESWT is an effective and noninvasive treatment method for mild to moderate carpal tunnel syndrome. Further studies with larger sample size and longer follow-up period are needed to verify the clinical efficacy of fESWT.

Author Contribution All authors read and approved the final version of the manuscript.

Funding Open access funding provided by Medical University of Vienna.

\section{Compliance with ethical guidelines}

Conflict of interest C. Gesslbauer, M. Mickel, O. Schuhfried, D. Huber, M. Keilani, and R. Crevenna certify that there is no conflict of interest with any financial organization regarding the material discussed in the manuscript.

Ethical standards All procedures followed were in accordance with the ethical standards of the responsible committee on human experimentation (institutional and national) and with the Helsinki Declaration of 1975, as revised in 2008.

Open Access This article is licensed under a Creative Commons Attribution 4.0 International License, which permits use, sharing, adaptation, distribution and reproduction in any medium or format, as long as you give appropriate credit to the original author(s) and the source, provide a link to the Creative Commons licence, and indicate if changes were made. The images or other third party material in this article are included in the article's Creative Commons licence, unless indicated otherwise in a credit line to the material. If material is not included in the article's Creative Commons licence and your intended use is not permitted by statutory regulation or exceeds the permitted use, you will need to obtain permission directly from the copyright holder. To view a copy of this licence, visit http://creativecommons.org/licenses/by/4.0/.

\section{References}

1. Padua L, Coraci D, Erra C, Pazzaglia C, Paolasso I, Loreti C, et al. Carpal tunnel syndrome: clinical features, diagnosis, and management. Lancet Neurol. 2016;15(12):1273-84. https://doi.org/10.1016/S1474-4422(16)30231-9.

2. Wipperman J, Goerl K. Carpal tunnel syndrome: diagnosis and management. Am Fam Physician. 2016;94(12):993-9.

3. O'Connor D, Marshall S, Massy-Westrop N. Non-surgical treatment (other than steroid injection) for carpal tunnel syndrome. Cochrane Database Syst Rev. 2003; https://doi. org/10.1002/14651858.CD003219.

4. Werner RA, Franzblau A, Gell N. Randomized controlled trial of nocturnal splinting for active workers with symptoms of carpal tunnel syndrome. Arch Phys Med Rehabil. 2005;86:1-7.

5. Lazovic M, Ilic-Stojanovic O, Kocic M, ZivkovicV, Hrkovic M, Radosavljevic N. Placebo-controlled investigation of lowlevel laser therapy to treat carpal tunnel syndrome. Photomed Laser Surg. 2014;32(6):336-44. https://doi.org/10. 1089/pho.2013.3563.

6. Wang CJ. An overview of shock wave therapy in musculoskeletal disorders. Chang Gung Med J. 2003;26(4):220-32.

7. Ke MJ, Chen LC, Chou YC, Li TY, Chu HY, Tsai CK, et al. The dose-dependent efficiency of radial shock wave therapy for patients with carpal tunnel syndrome: a prospective, randomized, single-blind, placebo-controlled trial. Sci Rep. 2016;6:38344. https://doi.org/10.1038/srep38344.

8. Schmitz C, Császár NB, Milz S, Schieker M, Maffulli N, Rompe JD, et al. Efficacy and safety of extracorporeal shock wave therapy for orthopedic conditions: a systematic review on studies listed in the PEDro database. Br Med Bull. 2015;116:115-38. https://doi.org/10.1093/bmb/ldv047.

9. Crevenna R, Mickel M, Keilani M. Extracorporeal shock wave therapy in the supportive care and rehabilitation of cancer patients. Support Care Cancer. 2019;27(11):4039-41. https://doi.org/10.1007/s00520019-05046-y.

10. HolfeldJ, Tepekoylu C, KozarynR, UrbschatA,ZacharowskiK, Grimm M, et al. Shockwave therapy differentially stimulates endothelial cells: implications on the control of inflammation via toll-like receptor 3 . Inflammation. 2014;37(1):65-70.

11. Kim JC, Jung SH, Lee SU, Lee SY. Effect of extracorporeal shockwave therapy on carpal tunnel syndrome: A systematic review and meta-analysis of randomized controlled trials. Medicine. 2019;98(33):e16870. https://doi.org/10. 1097/MD.0000000000016870.

12. Paoloni M, Tavernese E, Cacchio A, D'orazi V, Ioppolo F, Fini M, et al. Extracorporeal shock wave therapy and ultrasound therapy improve pain and function in patients with carpal tunnel syndrome. A randomized controlled trial. Eur J Phys Rehabil Med. 2015;51(5):521-8.

13. Mariotto S, Cavalieri E, Amelio E, Ciampa AR, de Prati AC, Marlinghaus E, et al. Extracorporeal shock waves: from lithotripsy to anti-inflammatory action by NO production. Nitric Oxide. 2005;12(2):89-96.

14. Seok H, Kim SH. The effectiveness of extracorporeal shock wave therapy vs. local steroid injection for management of carpal tunnel syndrome: a randomized controlled trial. Am JPhys Med Rehabil. 2013;92(4):327-34. https://doi.org/10. 1097/PHM.0b013e31826edc7b.

15. Weihs AM, Fuchs C, Teuschl AH, Hartinger J, Slezak P, Mittermayr R, et al. Shock wave treatment enhances cell proliferation and improves wound healing by ATP release-coupled extracellular signal-regulated kinase(ERK) 
activation. J Biol Chem. 2014;289(39):27090-104. https:// doi.org/10.1074/jbc.M114.580936.

16. Ohtori S, Inoue G, Mannoji C, Saisu T, Takahashi K, Mitsuhashi S, et al. Shock wave application to rat skin induces degeneration and reinnervation of sensory nerve fibres. Neurosci Lett. 2001;315(1-2):57-60.

17. Hausner T, Nógrádi A. The use of shock waves in peripheral nerve regeneration: new perspectives? Int Rev Neurobiol. 2013;109:85-98. https://doi.org/10.1016/B978-0-12420045-6.00003-1. Review.

18. MenseS, Hoheisel U. Shock wave treatment improves nerve regeneration in the rat. Muscle Nerve. 2013;47(5):702-10. https://doi.org/10.1002/mus.23631.

19. Xu D, MaW, JiangW, HuX, JiangF, Mao C, etal. Arandomized controlled trial: comparing extracorporeal shockwavetherapy versus local corticosteroid injection for the treatment of carpal tunnel syndrome. Int Orthop. 2020;44(1):141-6. https://doi.org/10.1007/s00264-019-04432-9.

20. Wu YT, Ke MJ, Chou YC, Chang CY, Lin CY, Li TY, et al. Effect of radial shock wave therapy for carpal tunnel syndrome: a prospective randomized, double-blind, placebocontrolled trial. J Orthop Res. 2016;34(6):977-84. https:// doi.org/10.1002/jor.23113.

21. Vahdatpour B, Kiyani A, Dehghan F. Effect of extracorporeal shock wave therapy on the treatment of patients with carpal tunnel syndrome. AdvBiomed Res. 2016;5:120. https://doi. org/10.4103/2277-9175.186983.

22. Notarnicola A, Maccagnano G, Tafuri S, Fiore A, Pesce V, MorettiB.Comparison of shockwave therapyandnutraceutical composed of Echinacea angustifolia, alpha lipoic acid, conjugated linoleic acid and quercetin (perinerv) in patients with carpal tunnel syndrome. Int J Immunopathol Pharmacol. 2015;28(2):256-62. https://doi.org/10.1177/ 0394632015584501.

23. Raissi GR, Ghazaei F, Forogh B, MadaniSP, DaghaghzadehA, Ahadi T. The effectiveness of radial Extracorporeal shock waves for treatment of carpal tunnel syndrome: a randomized clinical trial. Ultrasound Med Biol. 2017;43(2):453-60. https://doi.org/10.1016/j.ultrasmedbio.2016.08.022.Epub.

24. American Association of Electrodiagnostic Medicine, American Academy of Neurology, American Academy of Physical Medicine and Rehabilitation. Practice parameter for electrodiagnostic studies in carpal tunnel syndrome: summary statement. Muscle Nerve. 2002;25(6):918-22.

25. Bland JD. A neurophysiological grading scale for carpal tunnel syndrome. Muscle Nerve. 2000;23(8):1280-3.

26. Bijur PE, Silver W, Gallagher EJ. Reliability of the visual analog scale for measurement of acute pain. Acad Emerg Med. 2001;8:1153-7.
27. Härkönen R, Harju R, Alaranta H. Accuracy of the Jamar dynamometer. J Hand Ther. 1993;6(4):259-62.

28. Bullinger M. Erfassung der gesundheitsbezogenen Lebensqualität mit dem SF-36 Health Survey. Bundesgesundheitsblatt Gesundheitsforschung Gesundheitsschutz. 2000;(43):190-7.

29. KeilaniM,PernickaE,Paternostro-SlugaT,SychaT, SchettG, Pieber K, et al. Transkulturelle Adaptation einer deutschen Version des „Boston Carpal tunnel Syndrome Questionnaire" zum Einsatz bei Patienten mit Karpaltunnelsyndrom. Phys Med Rehab Kuror. 2007;17:A20. https://doi. org/10.1055/s-2007-99267.

30. Levine DW, Simmons BP, Koris MJ, Daltroy LH, Hohl GG, Fossel $\mathrm{AH}$, et al. A self-administered questionnaire for the assessment of severity of symptoms and functional status in carpal tunnel syndrome. J Bone Joint Surg Am. 1993 75(11):1585-92. https://doi.org/10.2106/00004623199311000-00002.

31. Ito K, Fukumoto Y, Shimokawa H. Extracorporeal shock wave therapy as a new and non-invasive angiogenic strategy. Tohoku JExp Med. 2009;219(1):1-9.

32. Stojadinovic A, Elster EA, Anam K, et al. Angiogenic response to extracorporeal shock wave treatment in murine skin isografts. Angiogenesis. 2008;11(4):369-80.

33. Takahashi N, Wada Y, Ohtori S, et al. Application of shock waves to rat skin decreases calcitonin generelated peptide immunoreactivity in dorsal root ganglion neurons. Auton Neurosci. 2003;107(2):81-4.

34. Mariotto S, de Prati AC, Cavalieri E, Amelio E, Marlinghaus E, Suzuki H. Extracorporeal shock wave therapy in inflammatory diseases: molecular mechanism that triggers antiinflammatory action. Curr Med Chem. 2009;16(19):236672. https://doi.org/10.2174/092986709788682119.

35. Chao Y-H, Tsuang Y-H, Jui-Sheng S, Chen L-T, Chiang Y-F, Wang C-C, et al. Effects of shock waves on tenocyte proliferation and extracellular matrix metabolism. Ultrasound Med Biol. 2008;34(5):841-52.

36. Berta L, Fazzari A, Ficco AM, Maurici Enrica P, Graziella Catalano M, Frairia R. Extracorporeal shock waves enhance normal fibroblast proliferation in vitro and activate mRNA expression for TGF-betal and for collagen types I and III. Acta Orthop. 2009;80(5):612-7.

37. Miyamoto H, Miura T, Morizaki Y, Uehara K, Ohe T, Tanaka S. Hand Surg. 2013;18(2):209-14.

Publisher's Note Springer Nature remains neutral with regard to jurisdictional claims in published maps and institutional affiliations. 